\title{
Vision-based system for line following mobile robot
}

\begin{abstract}
A vision based application is proposed for a line following mobile robot. A low-cost Webcam is used as the sensor and the image buffers are processed via a customized image segregation method to output necessary information for the mobile robot's controller under uncontrollable lighting condition. The task is to allow the mobile robot to navigate through a predefined path marked by a white line on a dark green floor surface. Experimental results shows that the mobile robot successfully able to navigate throughout the provided path. This technique is robust, reliable and easily modified for used in any other applications, such in industrial applications where costly sensors and transducers are normally used.
\end{abstract}

Keyword: Vision system; Line following; Mobile robot 